Artículo de Revisión

\title{
PÉPTIDO NATRIURÉTICO: BIOMARCADOR DE PRONÓSTICO EN ANESTESIA GERIÁTRICA
}

\author{
Dagoberto Ojeda D. ${ }^{1}$, Javiera Wurth O. ${ }^{2}$, Pablo Valenzuela L. ${ }^{3}$ y Renato Chacón A. ${ }^{1}$
}

Key words: Brain Natriurec Peptide, Aged 80 and over, Hip Fractures.

\section{Fisiopatología del envejecimiento}

El proceso de envejecimiento involucra múltiples cambios. Uno de los más cruciales es la disminución del consumo de oxígeno ${ }^{1}\left(\mathrm{VO}_{2}\right)$. $\mathrm{El} \mathrm{VO}_{2}$ máximo es la máxima cantidad de oxígeno que una persona puede usar realizando un ejercicio dinámico y es un reflejo del gasto cardiaco máximo. La disminución del consumo máximo de oxígeno comienza entre los 20-30 años y cae aproximadamente un $10 \%$ por década. Este fenómeno se atribuye a una disminución del metabolismo basal y de la masa de músculo esquelético. La disminución del $\mathrm{VO}_{2}$ de los ancianos va aparejada de una disminución del gasto cardiaco que también es provocada por diversas causas: disminución de la frecuencia cardiaca, menor respuesta adrenérgica, aumento de la presión arterial y por tanto aumento de la postcarga, disfunción diastólica que lleva a una disminución de la precarga. Todos estos fenómenos disminuyen el volumen expulsivo (Figura 1).

La disfunción diastólica es un cambio propio del envejecimiento ${ }^{1,2}$; a nivel del miocardio los miocitos son reemplazados por fibroblastos que producen colágeno y fibrosis intersticial. Esto, en conjunto con la pérdida de miocitos por necrosis, provoca que el ventrículo izquierdo se vuelva rígido y menos complaciente, de manera que el llene diastólico se ve dificultado, pasando a tomar más importancia la contracción de la aurícula izquierda

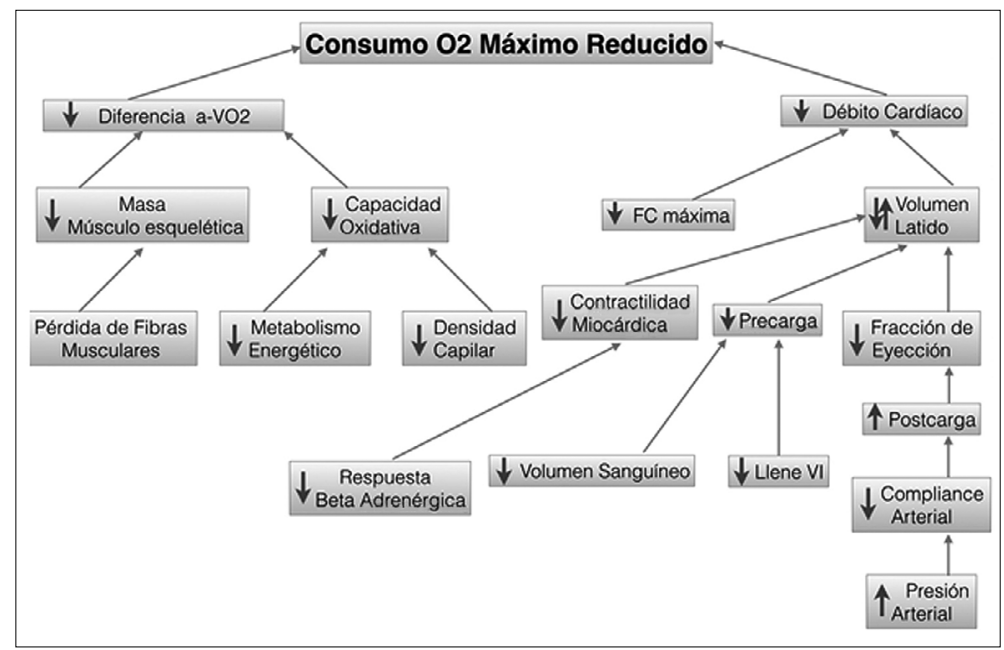

Figura 1. Reducción de la capacidad funcional cardiovascular y envejecimiento (Modificado de Helen O, Norman S. Cardiovascular Aging and heart failure. The European Journal of Heart Failure 2003; 5: 427-34).

Anestesiólogo Clínica Dávila.

Becada Anestesiología Universidad de Los Andes.

3 Médico Cirujano Universidad de Los Andes.

Los autores declaran no tener conflicto de interés. 
para conseguir el llene ventricular. En el miocardio, a nivel intracelular, se produce una disfunción de la bomba ATPasa del retículo sarcoplásmico que controla la recaptación de $\mathrm{Ca}^{++}$, esto lleva a una prolongación de la relajación iso volúmica. La disfunción diastólica, con el método de Doppler transmitral, tiene un patrón ecocardiográfico caracterizado por una mayor preponderancia de la onda A (referida a la contracción auricular), en relación a la onda $\mathrm{E}$ (onda de llenado rápido) (Figura 2).

El endurecimiento de la pared de las arterias aumenta la presión sistólica puesto que la energía de pulso generada por el corazón no es absorbida por la pared de una aorta rígida. Esta pérdida de elasticidad también provoca un enlentecimiento del flujo sanguíneo durante la diástole y una disminución de la presión diastólica con un aumento de la presión de pulso (Figura 3).

Todos estos cambios llevan a una disminución de la capacidad máxima de ejercitarse, lo que es más acentuado en mujeres que en hombres de similar edad y que se mide en MET o equivalente metabólico (Figura 4).

\section{Predictores}

La patología cardiovascular es la de mayor prevalencia en pacientes quirúrgicos ancianos. En Clínica Dávila estudiamos la cohorte de pacientes nonagenarios que habían sido operados entre los años 2001-2010 ${ }^{3}$ y encontramos que la prevalencia de patología cardiovascular era $60 \%$ (Figura 5). Roche $^{4}$, en pacientes ancianos que se operaban de fractura de cadera tuvo un $24 \%$ de comorbilidad cardiovascular, siendo ésta la más frecuente (Tabla 1). La presencia de patología cardiovascular es un factor de mal pronóstico en pacientes quirúrgicos

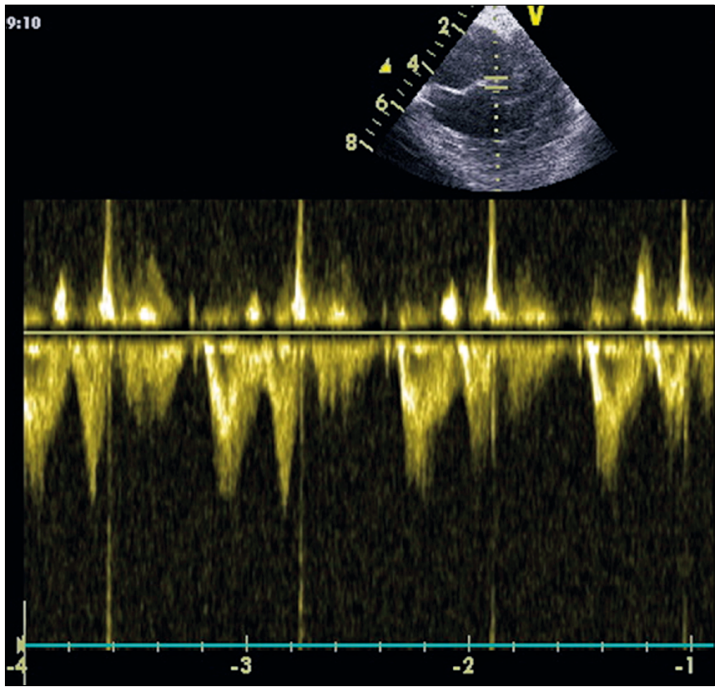

Figura 2. Doppler transmitral. Onda E y Onda A en disfunción diastólica (Gentileza Dr. Renato Chacón A. Anestesiología Clínica Dávila).

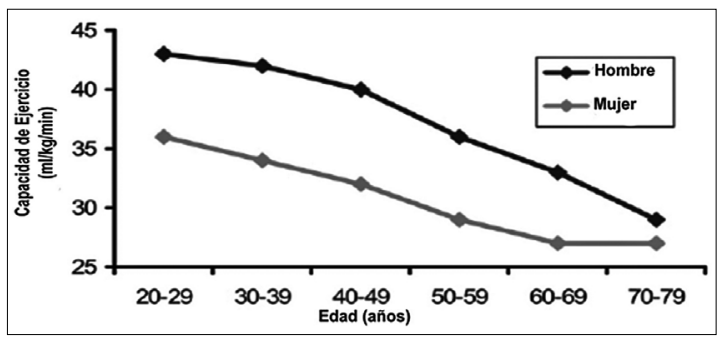

Figura 4. Disminución de la capacidad funcional y envejecimiento. (Modificado de Helen O, Norman S. Cardiovascular Aging and heart failure. The European Journal of Heart Failure. 2003; 5: 427-434).

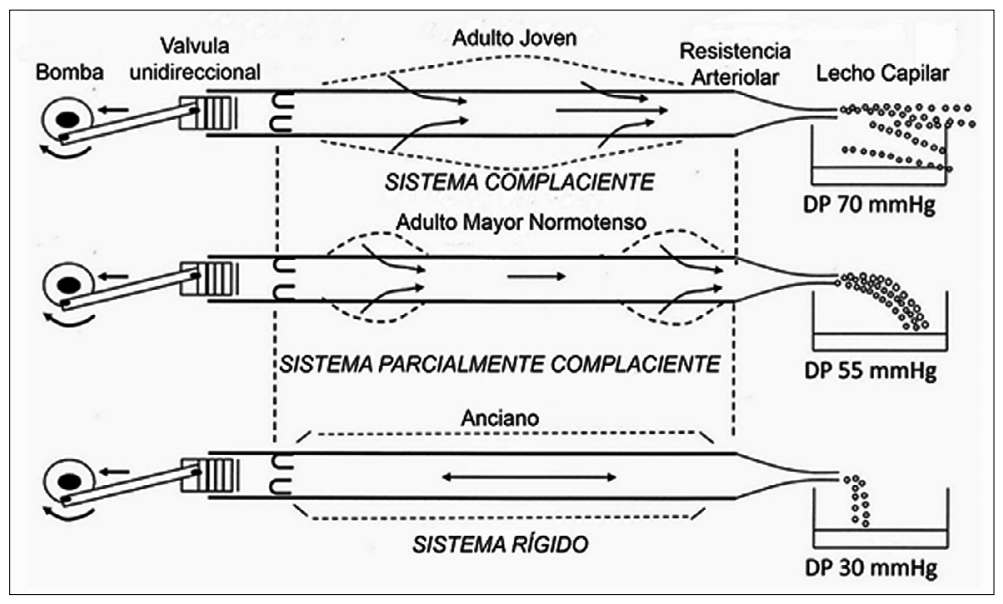

Figura 3. Distensibilidad aórtica, envejecimiento y flujo sanguíneo diastólico (Modificado de David S, Michael D, Leanne G. Diastolic Dysfunction, Cardiovascular Aging, and the Anesthesiologist. Anesthesiol Clin. 2009; 27: 497-517). 


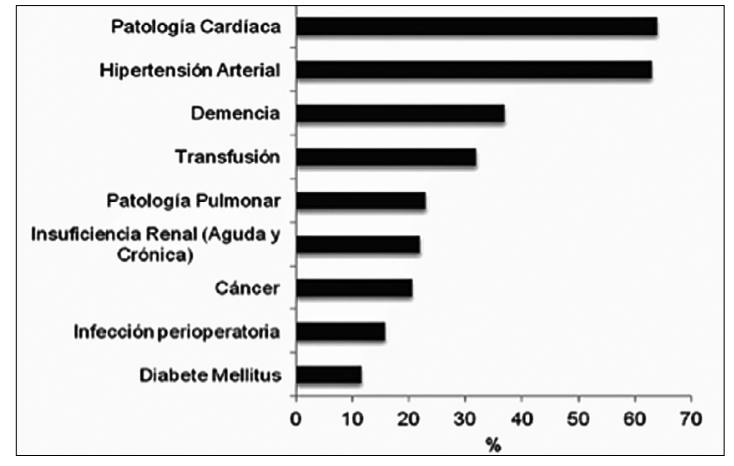

Figura 5. Prevalencia (\%) de patologías agregadas en pacientes nonagenarios. (Ojeda E, Gazabatt F. Sobrevida postoperatoria en pacientes nonagenarios. Estudio pronóstico en cohorte retrospectiva 2001-2010, Clínica Dávila. Rev Med Chile 2013; 141: 34-40).

ancianos, nuestros pacientes nonagenarios que eran portadores de patología cardiovascular tenían casi el doble de mortalidad que los que no la padecían. Roche también encontró que la presencia de insuficiencia cardiaca era un factor ominoso: 8 veces más mortalidad postoperatoria precoz (30 días) y 5 veces más mortalidad postoperatoria tardía (1 año plazo), que los no portadores de insuficiencia cardiaca.

En nuestro estudio de pacientes nonagenarios quirúrgicos ${ }^{3}$, la cirugía más prevalente fue la corrección de una fractura de cadera. Este tipo de cirugía adquiere el carácter de urgencia, puesto que existe evidencia ${ }^{5}$, si bien no es de primera categoría (metanálisis de estudios prospectivos y retrospectivos), que demuestra que la mortalidad a los 30 días y al año aumenta al retardar la cirugía más de 48 horas. De manera que estos pacientes muy ancianos, que probablemente estarán aquejados de patología cardiovascular deberían ser operados en un breve plazo que muchas veces no dará tiempo para completar todas las evaluaciones que se precisan para evitar consecuencias desastrosas. A esto se agrega el que la información clínica acerca de la capacidad funcional es poco clara o nula. Esto último no es menor; un estudio recientemente publicado ${ }^{6}$, demostró que el mejor predictor de complicaciones postoperatorias en general y cardiológicas en particular, eran los test de ejercicio con medición del $\mathrm{VO}_{2}$ (Figura 6), lo que nos lleva al inicio de esta revisión. En ancianos estas pruebas son difíciles de aplicar e imposibles si se trata de un paciente afectado de una fractura de cadera.

Existen algunas condiciones cardiológicas descompensadas que categóricamente requieren tratamiento urgente puesto que revisten una gravedad
Tabla 1. Comorbilidades en pacientes ancianos operados de fractura de cadera (Fuente: Roche. BMJ 2005; 331: 1374).

\begin{tabular}{|lr|}
\hline Comorbilidad & n (\%) \\
Enfermedad Cardiovascular & $597(24)$ \\
AVE & $322(13)$ \\
Enfermedad Respiratoria & $343(14)$ \\
Enfermedad Renal & $66(3)$ \\
Diabetes Mellitus & $232(9)$ \\
Enfermedad Reumatológica & $84(3)$ \\
Enfermedad de Parkinson & $97(4)$ \\
Enfermedad de Paget & $186(8)$ \\
Tabaquismo actual & $13(1)$ \\
Uso de Corticoides & $240(10)$ \\
$\mathrm{N}^{0}$ de Comorbilidades & \\
1 & $861(35)$ \\
2 & $414(17)$ \\
$\geq 3$ & $162(7)$ \\
\hline
\end{tabular}

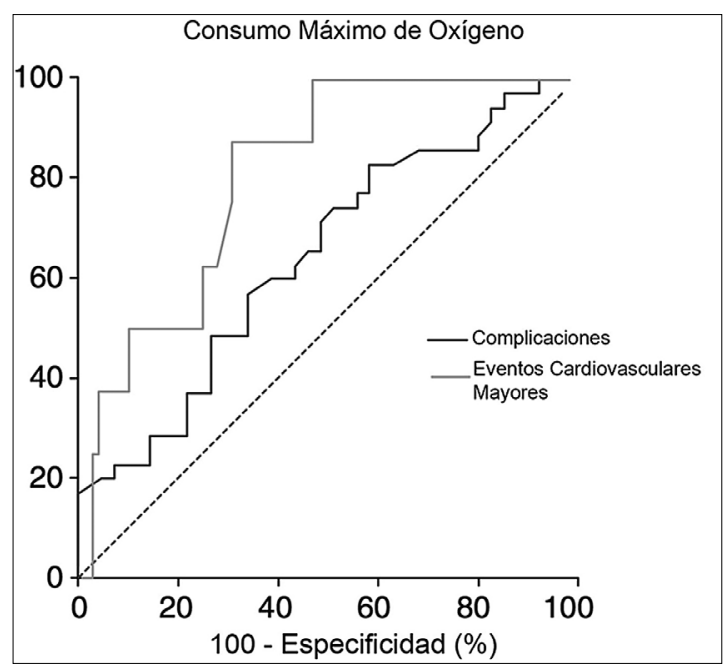

Figura 6. Consumo de Oxígeno y Eventos Cardiovasculares (Modificado de James S et al. Comparison of the prognostic accuracy of scoring systems, cardiopulmonary exercise testing, and plasma biomarkers: a singlecentre observational pilot study. Br J Anaesth 2014; 112 (3): 491-7).

mayor que la postergación de la cirugía ${ }^{7}$ (Tabla 2). Por otro lado existen otras patologías (Tabla 3), que aunque compensadas aumentan el riesgo peroperatorio (Tabla 4) de sufrir un evento cardiovascular ${ }^{8}$ severo (arritmia, infarto miocárdico, muerte), es 
Tabla 2. Condiciones cardiacas activas y/o descompensadas que requieren evaluación y tratamiento antes de cirugía no cardiaca (Clase I - Evidencia y/o acuerdo general de que un determinado procedimiento diagnóstico/tratamiento es beneficioso, útil y efectivo; Nivel de Evidencia B - Datos procedentes de un único ensayo clínico con distribución aleatoria o de grandes estudios sin distribución aleatoria)

\begin{tabular}{|ll|}
\hline Condición & Ejemplo \\
Síndromes coronarios inestables & Angina inestable \\
Insuficiencia cardiaca descompensada & NYHA Clase IV, empeoramiento de insuficiencia cardiaca de reciente \\
& comienzo \\
Arritmias severas & Bloqueo aurículo-ventricular grado 3 \\
& Bloqueo aurículo-ventricular Mobitz II \\
& Arritmias ventriculares sintomáticas \\
& Arritmias supra ventriculares con frecuencia ventricular $>100$ lpm en \\
& reposo \\
& Bradicardia sintomática \\
Taquicardia ventricular & Estenosis aórtica con gradiente media $>40$ mmHg, área valvular $<1$ \\
cm² o sintomática & Estenosis mitral sintomática: disnea progresiva con ejercicio, síncope \\
Enfermedad valvular severa & o insuficiencia cardiaca \\
\hline
\end{tabular}

Fuente: ACC/AHA Guidelines on Perioperative Cardiovascular Evaluation and Care. JACC 2007; 50: 159-242.

Tabla 3. Índice de riesgo cardiaco revisado

\begin{tabular}{|c|}
\hline Historia de angor pectoris \\
\hline Historia de infarto miocárdico \\
\hline Historia de insuficiencia cardiaca \\
\hline Historia de AVE/TIA \\
\hline Diabetes mellitus insulino-requiriente \\
\hline Insuficiencia renal $\left(\right.$ creatinina $>2 \mathrm{mg} \cdot \mathrm{dl}^{-1}$ o clearence creatinina $<60 \mathrm{ml} \bullet \mathrm{min}^{-1}$ ) \\
\hline $\begin{array}{l}\text { Fuente: European Heart Journal 2009; 30: 2769-2812. Guidelines for pre-operative cardiac risk assesment and perio- } \\
\text { perative cardiac management in non-cardiac surgery. }\end{array}$ \\
\hline
\end{tabular}

Tabla 4. Complicaciones cardiovasculares postoperatorias. (Modificado de Biccard \& Rodseth. Br J Anesth 2011; 107:133-43)

\begin{tabular}{|ccc|}
\hline $\mathbf{N}^{\mathbf{0}}$ factores de riesgo cardiaco & $\begin{array}{c}\text { Complicaciones CV mayores \% } \\
\text { (IC 95\%) }\end{array}$ & $\begin{array}{c}\text { Muerte CV } \\
\text { (\%) }\end{array}$ \\
0 & $0,5(0,2-1,1)$ & 0,3 \\
1 & $1,3(0,7-2,1)$ & 0,7 \\
2 & $3,6(2,1-5,6)$ & 1,7 \\
$\geq 3$ & $9,1(5,5-13,8)$ & 3,6 \\
\hline
\end{tabular}

$\mathrm{CV}$ : cardiovascular. 
Tabla 5. Riesgo cardiaco según tipo de cirugía no cardiaca: incidencia combinada de muerte e infarto miocárdico

\begin{tabular}{|ll|}
\hline Categoría de Riesgo & Ejemplos \\
Alto $(>5 \%)$ & Cirugía aórtica y vascular mayor \\
Intermedio $(1-5 \%)$ & Cirugía vascular periférica \\
& Cirugía Intraperitoneal \\
& Cirugía Intratorácica \\
& Endarterectomía carotídea \\
Bajo $(<1 \%)$ & Cirugía de cabeza y cuello \\
& Cirugía prostática \\
& Procedimientos superficiales \\
& Procedimientos endoscópicos \\
& Cirugía cataratas \\
& Cirugía de mama \\
\hline
\end{tabular}

Fuente: ACC/AHA Guidelines on Perioperative Cardiovascular Evaluation and Care JACC 2007; 50: 159-242.

Tabla 6. Razones para retardar la cirugía de una fractura de cadera

\begin{tabular}{|c|c|}
\hline Aceptable & Inaceptable \\
\hline Hemoglobina $<8 \mathrm{~g} \bullet \mathrm{dL}^{-1}$ & Falta de pabellón \\
\hline $\begin{array}{l}\mathrm{Na}^{+} \text {plasmático }<120 \text { ó }>150 \mathrm{mmol} \cdot \mathrm{L}^{-1} \\
\mathrm{~K}^{+} \text {plasmático }<2,8 \text { ó }>6,0 \mathrm{mmol} \cdot \mathrm{L}^{-1}\end{array}$ & Espera resultado de una ecocardiografía \\
\hline Diabetes descompensada & Disponibilidad de traumatólogo \\
\hline $\begin{array}{l}\text { Insuficiencia ventricular Izquierda descompensada o de } \\
\text { reciente comienzo }\end{array}$ & Alteraciones electrolíticas menores \\
\hline \multicolumn{2}{|l|}{ Arritmia ventricular con frecuencia ventricular $>120 \mathrm{lpm}$} \\
\hline \multicolumn{2}{|l|}{ Infección pulmonar con sepsis } \\
\hline Coagulopatía reversible & \\
\hline
\end{tabular}

Modificado de Griffiths et al. Management of proximal femoral fractures 2011. Anaesthesia 2012; 67: 85-98.

lo que se llama índice de riesgo cardíaco revisado (RCRI).

Además, existen procedimientos quirúrgicos que se acompañan de un mayor riesgo de complicaciones cardiacas, estando la cirugía de fractura de cadera en la categoría intermedia (Tabla 5).

Griffiths ${ }^{9}$, en las guías clínicas de fractura de cadera del Reino Unido encuentra causas muy justificadas para retrasar la cirugía de una fractura de cadera en ancianos, sin embargo, la espera para realizar una ecocardiografía no es una de ellas (Tabla $6)$.

\section{Biomarcadores}

Habiendo reconocido un problemas como es el de tener un paciente muy lábil, casi sin posibilidades de evaluar su capacidad funcional, sometido a una cirugía que no es conveniente retardar a no ser por causas muy justificadas, entran en juego los biomarcadores. Estos son un conjunto de estructuras biológicas: proteínas, hormonas, enzimas, que nos entregan rápidamente una información que no es proporcionada por la clínica y que por tanto nos ayudan a tomar decisiones "al lado de la cama del paciente". Idealmente, la relación costo/beneficio de los biomarcadores debería ser eficiente. La utilidad de los biomarcadores ${ }^{10}$ puede apreciarse en la (Tabla 7).

Los biomarcadores cardiacos nos orientan acerca del stress, disfunción e injuria del miocito miocárdico. Dentro de ellos, el péptido natriurético destaca por ser liberado desde el miocito car- 
Tabla 7. Utilidad de los biomarcadores. (Modificado de Ray, Anesthesiology 2010; 112)

\begin{tabular}{|c|c|c|}
\hline Rol & Descripción & Ejemplos \\
\hline Diagnóstico de una enfermedad & $\begin{array}{l}\text { Hacer el diagnóstico más seguro, } \\
\text { expedito y barato que los métodos } \\
\text { habituales }\end{array}$ & $\begin{array}{l}\text { Troponina Ic en el diagnóstico del } \\
\text { infarto miocárdico }\end{array}$ \\
\hline Evaluación de la gravedad & $\begin{array}{l}\text { Identificar a un subgrupo de pacientes } \\
\text { con una forma severa de la enferme- } \\
\text { dad con mayor probabilidad de muer- } \\
\text { te o complicaciones severas }\end{array}$ & $\begin{array}{l}\text { Procalcitonina identifica pacientes } \\
\text { con sepsis con mal pronóstico } \\
\text { Troponina Ic identifica mal pronós- } \\
\text { tico en TEP }\end{array}$ \\
\hline Evaluación del riesgo & $\begin{array}{l}\text { Identificar a un subgrupo de pacientes } \\
\text { que pueden sufrir complicaciones } \\
\text { severas después de una intervención }\end{array}$ & $\begin{array}{l}\text { BNP y complicaciones postoperato- } \\
\text { rias en cirugía no-cardiaca }\end{array}$ \\
\hline Predecir el efecto de una droga & $\begin{array}{l}\text { Identificar la respuesta farmacológica } \\
\text { de un paciente (eficacia, toxicidad, } \\
\text { farmacocinética) }\end{array}$ & Eficacia del clopidogrel \\
\hline Monitorización & Evaluar respuesta terapéutica & $\begin{array}{l}\text { Procalcitonina y duración de antibió- } \\
\text { tico terapia }\end{array}$ \\
\hline
\end{tabular}

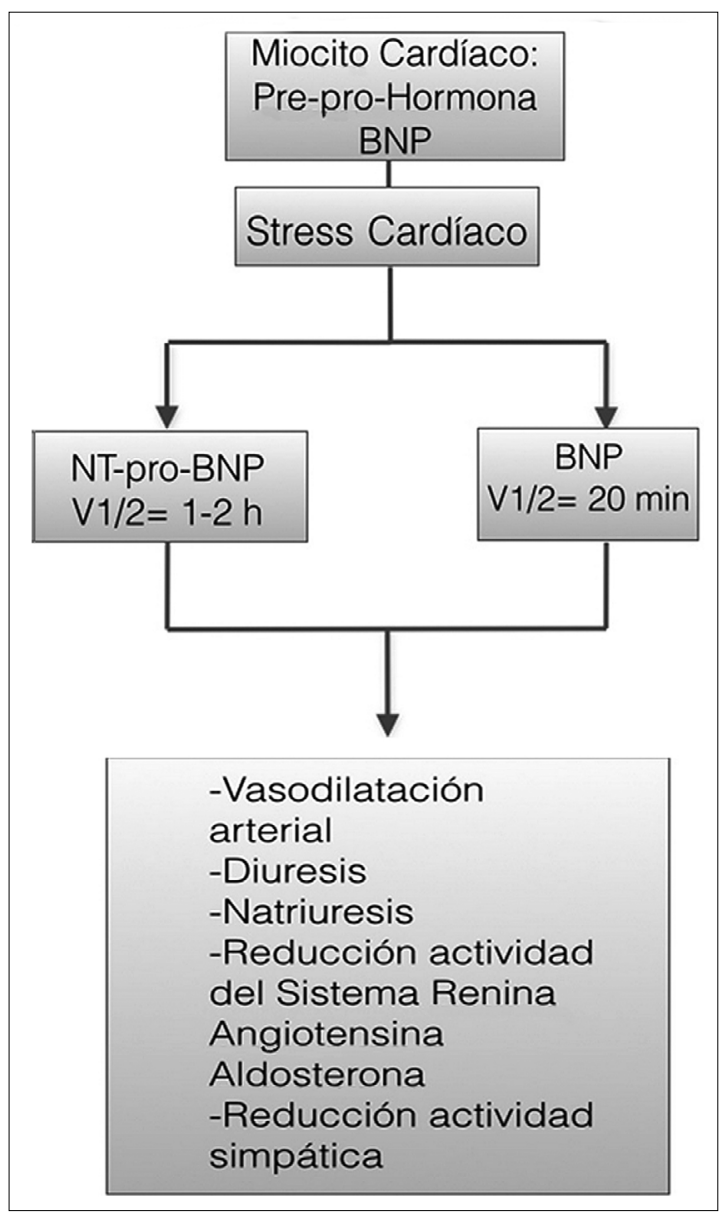

Figura 7. Péptido Natriurético: Efectos. diaco en condiciones de aumento de la tensión del ventrículo izquierdo por sobrecarga de presión o de volumen ${ }^{11}$. El precursor es fragmentado en dos derivados: fracción aminoterminal del péptido natriurético tipo B (NT-proBNP, acrónimo del inglés NT-pro-Brain Natriuretic Peptide) y Péptido Natriurético Cerebral (BNP, acrónimo del inglés Brain Natriuretic Peptide), el primero de vida media más larga y el último más corta (Figura 7). El efecto de estos péptidos es opuesto a los de la insuficiencia cardiaca (Figura 8). Estas características del péptido natriurético hicieron pensar que pudiera utilizarse como un biomarcador de insuficiencia cardiaca y a su vez, como la insuficiencia cardiaca es un índice ominoso asociado a complicaciones, se buscó utilidad como marcador de sobrevida y eventos cardiacos severos postoperatorios.

Kistorp ${ }^{12}$, observó en una cohorte de pacientes entre 50 y 89 años que la sobrevida a los 5 años era significativamente menor en aquellos que los niveles de NT-proBNP eran mayores de $411 \mathrm{pg} \cdot \mathrm{ml}^{-1}$. Curthbertson $^{13}$ a su vez, encontró en 40 pacientes con una edad mediana de 74 años sometidos a cirugía de urgencia mayor (no cardiaca), que la mortalidad e injuria miocárdica se asociaban a niveles preoperatorios más elevados de BNP. La predicción que se podía hacer con estos resultados, era excelente: con un área bajo la curva ROC de 0,85 $(0,72-0,98)$. Koç ${ }^{14}$, en un grupo de pacientes con insuficiencia cardiaca observó una correlación inversa estadísticamente significativa $(p=0,004)$, entre niveles de NT-proBNP y fracción de eyección por ecocardiografía. Vale decir, a menor fracción de 


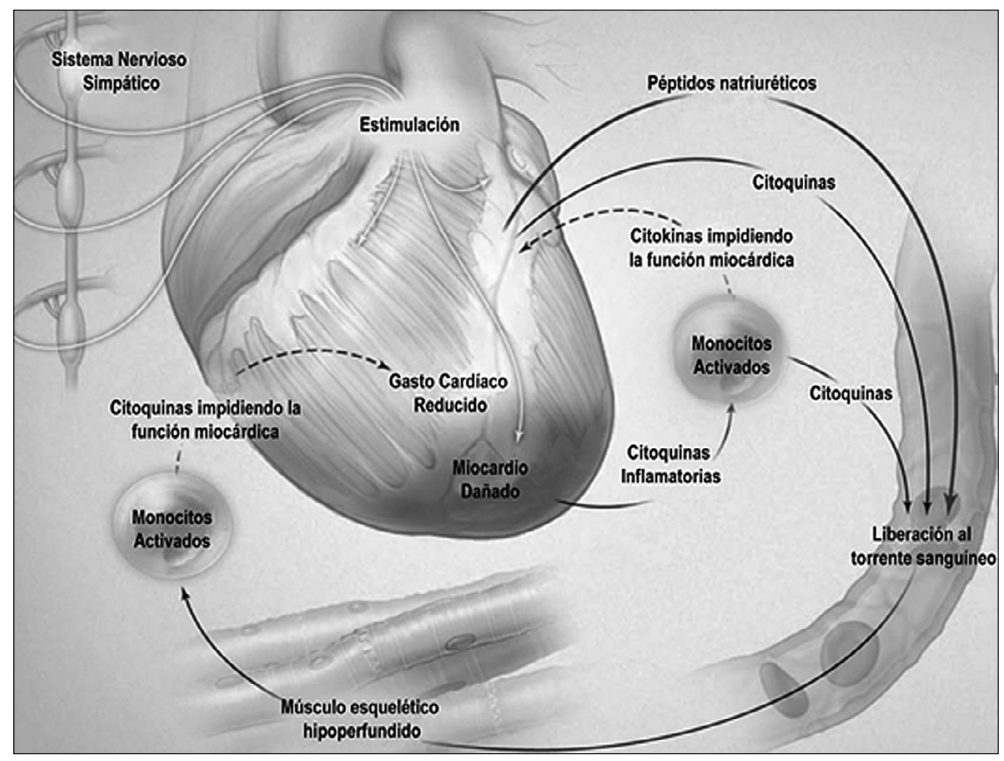

Figura 8. Péptido natriurético en insuficiencia cardiaca. (Modificado de Braunwald et al. Biomarker in Heart Failure. N Engl Med 2008; 358: 2148 59).

Tabla 8. Valor predictivo aditivo para MACE (eventos adversos cardiovasculares severos) al combinar el Índice Revisado de Riesgo Cardiaco y Biomarcadores

\begin{tabular}{|lcc|}
\multicolumn{1}{c}{ Método Predictivo } & AUC (p) & IC 95\% \\
RCRI & $0,592(0,019)$ & $0,570-0,615$ \\
RCRI+PCR & $0,694(0,019)$ & $0,673-0,715$ \\
RCRI+BNP & $0,735(0,018)$ & $0,714-0,754$ \\
RCRI+PCR+BNP & $0,772(0,017)$ & $0,752-0,790$ \\
\hline
\end{tabular}

RCRI: Índice de riesgo cardiaco revisado; PCR: Proteína C Reactiva; BNP: Brain Natriurec Peptide.

eyección, mayores eran los niveles de NT-proBNP.

También en la población general se ha visto que el BNP tiene un valor pronóstico. Linssen ${ }^{15}$, encontró que niveles más elevados de NT-proBNP se asociaban a eventos cardiovasculares y mortalidad por todas las causas pero no eran predictores de mortalidad no cardiovascular.

Biccard $^{16}$ investigó si el BNP permitía mejorar la predicción del riesgo cardiovascular peroperatorio obtenida del índice de riesgo cardiaco revisado en pacientes sometidos a cirugía vascular. Encontró que el BNP permitía reclasificar correctamente a los pacientes de riesgo intermedio. Esta reclasificación corregía la predicción de elevación de la troponina postoperatoria en más del $50 \%$ de los pacientes de riesgo intermedio, que son los pacientes en quienes es más difícil tomar una decisión. Esta mejoría de la predicción al incorporar el BNP, se evidenciaba por un aumento del área bajo la curva $\mathrm{ROC}^{7}$ desde 0,592 [0,570-0,615] al utilizar sólo el índice de riesgo, hasta $0,735[0,714-0,754]$ al agregar BNP, vale decir, se pasaba de una pobre predicción a una aceptable (Tabla 8). Choi $^{17}$ también comprobó una mejoría en la predicción de eventos cardiovasculares peroperatorios al incorporar el BNP al índice de riesgo cardiaco (Figura 9).

Oscarsson ${ }^{18}$, en una muestra de 69 pacientes ancianos sometidos a cirugía de fractura de cadera encontró que niveles $>3.984 \mathrm{ng} \cdot \mathrm{L}^{-1}$ de NT-ProBNP, eran predictores independientes (análisis multivariado), de eventos cardiacos postoperatorios: Odds Ratio 3,5 [1,3-9,5]. Curiosamente la insuficiencia cardiaca, que es parte del índice de riesgo cardiaco, no era un predictor significativo en forma multivariada (Tabla 9).

Messer ${ }^{19}$, en 98 pacientes sometidos a cirugía vascular y ortopédica, observó que niveles elevados de NT-ProBNP se asociaban a fracciones de eyección menores a $45 \%$, con una buena capacidad predictiva; un área bajo la curva ROC de 0,85 (Tabla 10).

Todos estos trabajos fueron realizados con gru- 


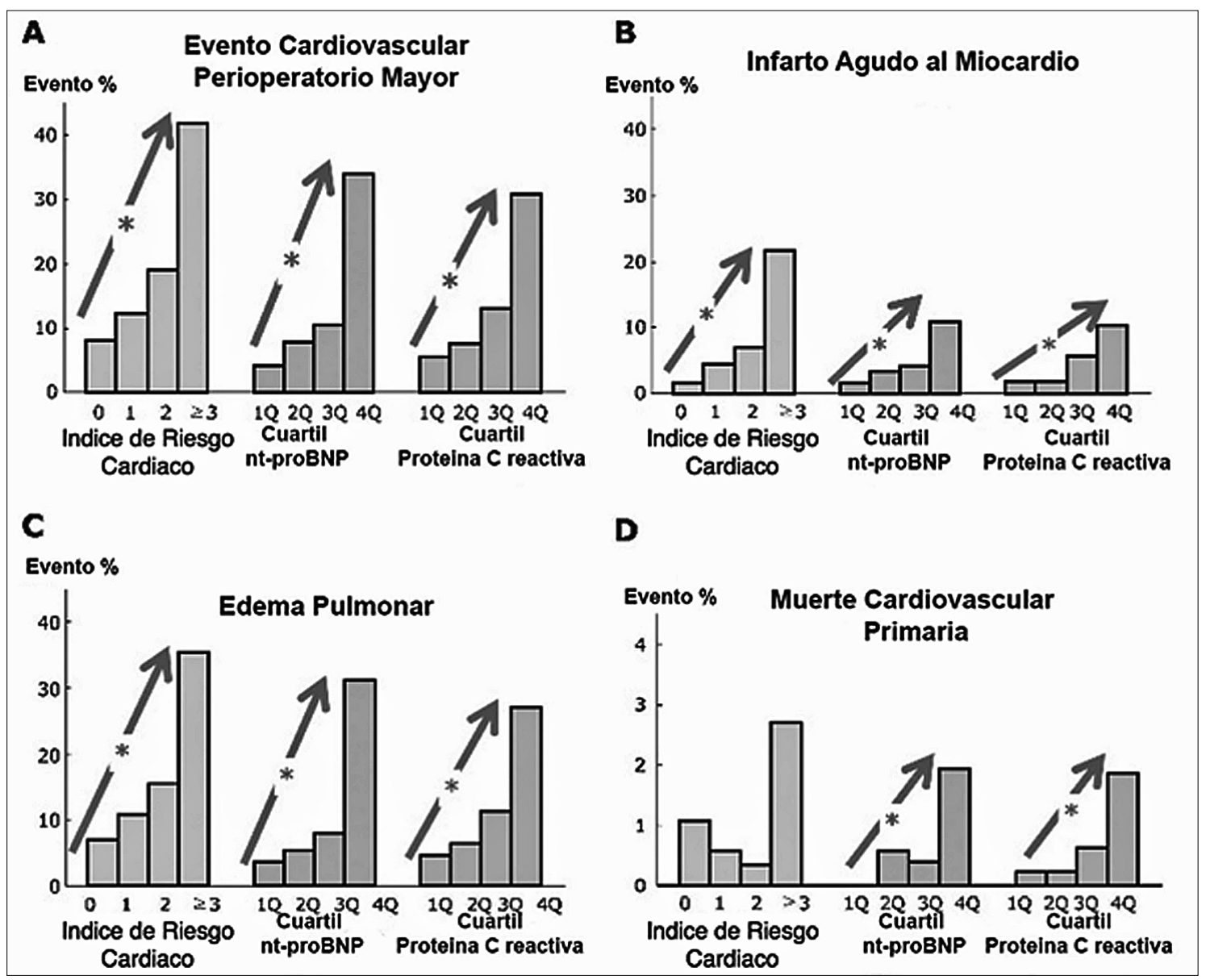

Figura 9. Péptido natriurético y proteína C reactiva en la predicción de eventos cardiovasculares postoperatorios. (Modificado de Choi et al. Heart 2010; 96: 56-62).

Tabla 9. Predictores de MACE (eventos adversos cardiovasculares severos) postoperatorios en pacientes ancianos operados de fractura de cadera. Modificado de Oscarsson Br J Anesth 2009; 103: 206

\begin{tabular}{|c|c|c|c|}
\hline & OR & IC $95 \%$ & p \\
\hline \multicolumn{4}{|l|}{ Univariado } \\
\hline NT-proBNP $>3.984 \mathrm{ng} \cdot \mathrm{L}^{-1}$ & 3,0 & $1,0-8,9$ & 0,05 \\
\hline Insuf. cardiaca congestiva & 3,0 & $1,0-9,0$ & 0,05 \\
\hline \multicolumn{4}{|l|}{ Multivariado } \\
\hline NT-proBNP > $3.984 \mathrm{ng} \cdot \mathrm{L}^{-1}$ & 3,5 & $1,3-9,5$ & 0,01 \\
\hline Edad & 1,1 & $1,0-1,2$ & 0,03 \\
\hline Insuf. cardiaca congestiva & 2,4 & $0,9-6,6$ & 0,08 \\
\hline Creatinina & 1,0 & $1,0-1,0$ & 0,09 \\
\hline
\end{tabular}

pos pequeños de pacientes y en la mayoría de los casos no hay cálculo de tamaño muestral ni del poder estadístico, por lo que sus conclusiones pudie- ran ser discutibles.

Existen metanálisis (Karthikeyan ${ }^{20}$, Ryding ${ }^{21}$ ) respecto a la utilidad del BNP como predictor de 
Tabla 10. Función ventricular y niveles de NT-proBNP. (Modificado de Messer, Anaesthesia 2008; 63: 482-7)

\begin{tabular}{|c|c|c|c|}
\hline & $\begin{array}{c}\text { Función ventricular } \\
\text { Normal } \\
n=75\end{array}$ & $\begin{array}{c}\text { Función ventricular } \\
\text { Anormal } \\
n=23\end{array}$ & $\begin{array}{c}\text { Total } \\
\mathrm{n}=\mathbf{9 8}\end{array}$ \\
\hline NT-proBNP pmol $\cdot \mathrm{L}^{-1}$ & $32,8(1,3-1141)$ & $131(39-1.174)$ & $52,4(1,3-1.174)$ \\
\hline
\end{tabular}

Tabla 11. Recomendaciones acerca del uso de BNP (Modificado de Poldermans, Eu J Anaesth 2010; 27: 92-137)

\begin{tabular}{|lcc|}
\hline \multicolumn{1}{|c|}{ Recomendación } & Clase & Nivel \\
$\begin{array}{l}\text { NT-proBNP y BNP deberían utilizar- } \\
\text { se como biomarcadores independien- } \\
\text { tes de MACE postoperatorios precoz } \\
\text { y tardío en pacientes de alto riesgo }\end{array}$ & IIa & B \\
$\begin{array}{l}\text { No se recomienda su uso rutinario } \\
\text { para predecir MACE postoperatorios }\end{array}$ & III & C \\
\hline
\end{tabular}

MACE: Evento adversos cardiovasculares severos; Clase IIa: El peso de la evidencia está a favor de la utilidad/ eficacia; Clase III: Evidencia o acuerdo general de que el tratamiento no es útil/efectivo y en algunos casos puede ser perjudicial; Nivel B: Datos procedentes de un único ensayo clínico con distribución aleatoria o de grandes estudios sin distribución aleatoria; Nivel C: Consenso de opinión de expertos y/o pequeños estudios, práctica habitual.

Tabla 12. Causas de elevación de BNP distintas de insuficiencia cardiaca descompensada. Modificado de Rodseth et al. B type natriureticapeptide - a diagnostic breakthrough in perioperative cardiac risk assesment. Anaesthesia 2009; 64: $165-178$

\begin{tabular}{|c|c|c|}
\hline Cardiaca & Pulmonar & Otras \\
\hline $\begin{array}{l}\text { Miocardiopatías: } \\
\text { - Miocardiopatía aguda } \\
\text { - Miocarditis } \\
\text { - Miocardiopatía hipertrófica }\end{array}$ & $\begin{array}{l}\text { Neumonía/Bronquitis } \\
\text { EPOC } \\
\text { Cáncer pulmonar } \\
\text { TEP }\end{array}$ & $\begin{array}{l}\text { Anemia } \\
\text { Enf. gastrointestinal } \\
\text { Cáncer } \\
\text { Pacientes críticos }\end{array}$ \\
\hline $\begin{array}{l}\text { Arritmias } \\
\text { Fibrilación auricular } \\
\text { Flutter auricular }\end{array}$ & Hipertensión pulmonar & Shock séptico \\
\hline Síndr. coronario agudo & Síndrome de distress respiratorio & $\begin{array}{l}\text { Quemaduras } \\
\text { AVE isquémico }\end{array}$ \\
\hline Valvulopatía & & $\begin{array}{l}\text { Apnea del sueño } \\
\text { Hipotiroidismo } \\
\text { Obesidad } \\
\text { Insuficiencia renal } \\
\text { Edad }\end{array}$ \\
\hline
\end{tabular}

eventos cardiovasculares peroperatorios, pero que combinan principalmente estudios observacionales, lo que se evidencia por la presencia de heterogeneidad significativa $(\mathrm{p}<0,05)$. En palabras simples, esto quiere decir que los estudios incluidos en su gran mayoría no son ensayos clínicos y, por tanto, con diferencias entre los pacientes estudiados que pudieran llevar a conclusiones sesgadas. Teniendo esto en mente, lo que se concluye de estos meta- nálisis es que el BNP es un predictor significativo de eventos cardiovasculares, mortalidad de origen cardiovascular y de toda causa, en el postoperatorio precoz ( $<30$ días) y a largo plazo ( $>6$ meses).

La evidencia disponible hasta el momento ${ }^{22}$ sugiere que el BNP debería ser utilizado como biomarcador pronóstico independiente en el tamizaje de pacientes de alto riego de eventos cardiacos perioperatorios. Su uso rutinario en todos los pacien- 
Tabla 13. Niveles de BNP para el diagnóstico de insuficiencia cardiaca. (Modificado de Rodseth et al. B type natriureticapeptide - a diagnostic breakthrough in perioperative cardiac risk assesment. Anaesthesia 2009; 64: 165-178)

\begin{tabular}{|c|c|c|c|}
\hline Insuficiencia cardiaca aguda & BNP & \multicolumn{2}{|c|}{ NT-proBNP } \\
\hline Inclusión & $>400 \mathrm{pg} \cdot \mathrm{ml}^{-1}$ & $\begin{array}{c}<50 \text { años } \\
50 \text { - } 75 \text { años } \\
>75 \text { años } \\
\text { I Renal Cr Severa }\end{array}$ & $\begin{array}{l}>450 \mathrm{pg} \bullet \mathrm{ml}^{-1} \\
>900 \mathrm{pg} \cdot \mathrm{ml}^{-1} \\
>1.800 \mathrm{pg} \bullet \mathrm{ml}^{-1} \\
>1.200 \mathrm{pg} \bullet \mathrm{ml}^{-1}\end{array}$ \\
\hline Exclusión & $<100 \mathrm{pg} \cdot \mathrm{ml}^{-1}$ & \multicolumn{2}{|c|}{$<300 \mathrm{pg} \cdot \mathrm{ml}^{-1}$} \\
\hline
\end{tabular}

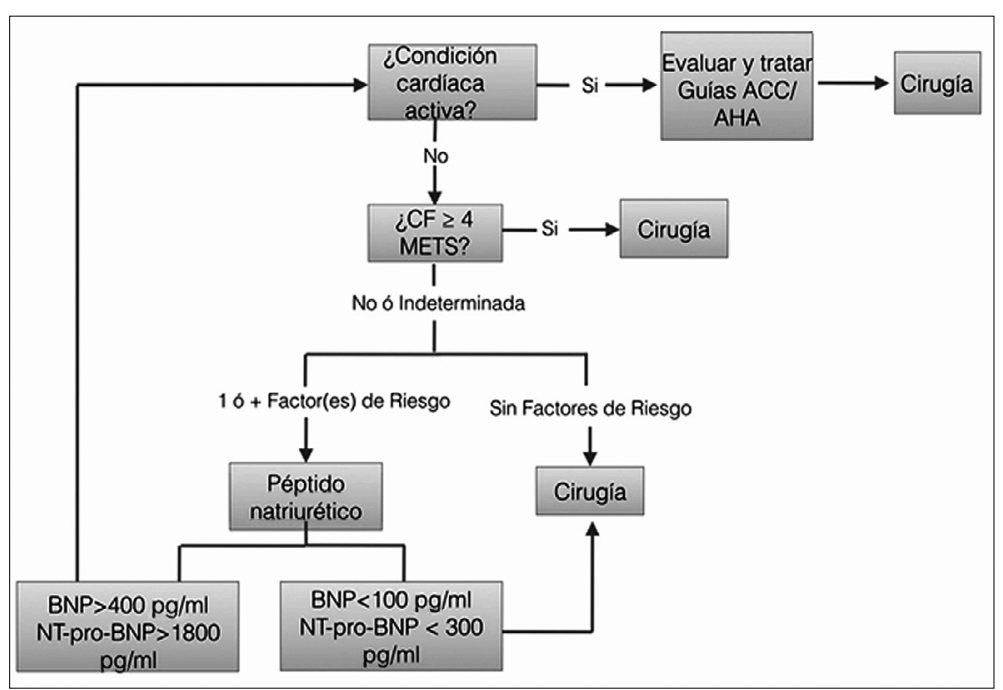

Figura 10. BNP en evaluación de pacientes ancianos sometidos a cirugía de fractura de cadera. Modificado de Siu et al., Osteoporos Int 2010; 21 (Suppl 4): S587-S591. tes quirúrgicos no estaría recomendado (Tabla 11).

$\mathrm{Si}$ se decide incorporar al BNP como parte de nuestro índice de riesgo cardiaco deben tomarse en cuenta otras causas de elevación de éste ${ }^{23}$, además de la insuficiencia cardiaca (Tabla 12). La edad y la presencia de insuficiencia renal también influyen ${ }^{23}$ (Tabla 13). Además debe considerarse la vida me- dia del péptido natriurético de que dispongamos, dado que el BNP tiene una vida media más corta que el NT-ProBNP.

Por último, el siguiente diagrama (adaptado de $\mathrm{Siu}^{24}$ ), pretende ilustrar el uso del BNP en pacientes ancianos sometidos a cirugía de fractura de cadera (Figura 10).

\section{REFERENCIAS}

1. Oxenham H, Sharpe N. Cardiovascular aging and heart failure. Eur $\mathbf{J}$ Heart F 2003; 5: 427-434

2. Sanders D, Dudley M, Groban L. Diastolic Dysfunction, Cardiovascular Aging and the Anesthesiologist. Anesthesiol Clin 2009; 27 : 497-517.

3. Ojeda D, Gazabatt F, Cisternas P, et al. Sobrevida Postoperatoria en Pacientes Nonagenarios. Estudio Pronóstico en Cohorte Retrospectiva 2001-2010, Clínica Dávila. Rev Med Chile 2013; 141: 34-40.

4. Roche JJW, Wenn RT, Sahota
O, et al. Effects of comorbidities and postoperative complications on mortality after hip fracture in elderly people: prospective observational cohort study. BMJ 2005; 331: 1374

5. Shiga T, Wajima Z, Ohe Y. Is operative delay associated with increased mortality of hip fractures patients? Systematic review, metaanalysis and meta-regression. Can J Anesth 2008; 55: 146-154.

6. James S, Jhanji S, Smith A, et al. Comparison of the prognosis accuracy of scoring systems, cardiopulmonary exercise testing, and plasma biomarkers: a single center observational pilot study. $\mathrm{Br} \mathrm{J}$ Anaesth 2014; 112: 491-497.

7. ACC/AHA Guidelines on Perioperative Cardiovascular Evaluation and Care. JACC 2007; 50: 159-242.

8. Biccard BM, Rodseth RN. Utility of risk predictors for preoperative cardiovascular risk prediction. $\mathrm{Br} \mathrm{J}$ Anaesth 2011; 107: 133-143.

9. Griffiths R, Alper J, Beckingsale A, et al. Guidelines. Management for proximal femoral fractures 2011. Anaesthesia 2012; 67: 85-98.

10. Ray P, Le Manach Y, Riou B, et al. Statistacal Evaluation of a Biomarker. Anesthesiology 2010; 112: 1023-1040. 
11. Braunwald E. Biomarkers in Heart Failure. N Engl J Med 2008; 385: 2148-2159.

12. Kistorp C, Raymond I, Pedersen F, et al. N-Terminal-Pro-Brain Natriuretic Peptide, C-Reactive Protein, and Urinary Albumin Levels as predictors of Mortality and Cardiovascular Events in Older Adults. JAMA 2005; 293: 1609-1616.

13. Curthbertson BH, Amiri AR, Croal BL, et al. Utility of B-type natriuretic peptide in predicting perioperative cardiac events in patients undergoing major non-cardiac surgery. Br J Anaesth 2007; 62: 875-881.

14. Koç M, Bozkurt A, Sahin DY, et al. Cut-off values of NT-proBNP for the prediction of low functional capacity, decreased ejection fraction and cardiovascular events in patients with heart failure. Cardiology Journal 2009; 16: 43-51.

15 Linssen GCM, Bakker SJL, Voors AA, et al. N-terminal pro-B-type natriuretic peptide is an independent predictor of cardiovascular morbidity and mortality in the general population. Eur Heart $\mathrm{J}$ 2010; 31: 120-127.

16. Biccard BM, Naidoo P. The role of brain natriuretic peptide in diagnostication and reclassification of risk in patients undergoing vacular surgery Anaesthesia 2011; 66: 379385.

17. Choi JH, Cho DK, Song YB, et al. Preoperative NT-proBNP and CRP predict perioperative major cardiac events in non-cardiac surgery. Heart 2010; 96: 56-62.

18. Oscarsson A, Fredrikson M, Sörliden $\mathrm{M}$, et al. N-terminal fragments of pro-B-type Natriuretic peptide is a predictor of cardiac events in high-risk patients undergoing hip fracture surgery. Br J Anaesth 2009; 103: 206-212.

19. Messer PB, Singh R, McAuley FT, et al. The use of N-terminal pro-B type Natriuretic peptide in a preoperative setting to predict left ventricular systolic Dysfunction on echocardiogram. Anaesthesia 2008; 63: 482-487.
20. Karthikeyan G, Moncur RA, Levine $\mathrm{O}$, et al. Is a pre-operative Brain Natriuretic Peptide or N-Terminal Pro-B-Type Natriuretic Peptide Measurement an independent predictor of adverse cardiovascular Outcomes within 30 days of noncardiac surgery? JACC 2009; 54 : 1599-1606.

21. Ryding ADS, Kumar S, Worthinton AM, et al. Prognostic value of Brain Natriuretic peptide in noncardiac surgery. A Metanalysis. Anesthesiology 2009; 111: 309-311.

22. Guidelines for pre-operative cardiac risk assessment and perioperative management in non-cardiac surgery. Eur J Anaesth 2010; 27; 92-137.

23. Rodseth RN. B type Natriuretic peptide- a diagnostic breakthrough in peri-operative risk assessment? Anaesthesia 2009; 64: 165-172.

24. Siu CW, Sun NCH, Lau TW, et al. Preoperative cardiac risk assessment in geriatrics patients with hip fractures: an orthopedic surgeon's perspective. Osteoporos Int 2010; 21: Supp(4) 587-591.
Correspondencia a:

Dagoberto Ojeda D.

eojedadinamarca@gmail.com 